Author's copy. Appeared in: Nørskov, M., Seibt J., Quick O. 2020. Culturally Sustainable Social Robotics-Proceedings of Robophilosophy 2020. Series Frontiers of AI and Its Applications, IOS Press, Amsterdam

\title{
A Horizontal Approach to Communication for Human-Robot Joint Action: Towards Situated and Sustainable Robotics

\author{
Kathleen BELHASSEIN ${ }^{\mathrm{ab}}$, Víctor FERNÁNDEZ CASTRO ${ }^{\text {ac,1 }}$ and Amandine \\ MAYIMA $^{\text {a }}$ \\ ${ }^{a}$ LAAS-CNRS, Université de Toulouse, CNRS, Toulouse, France. \\ ${ }^{\mathrm{b}}$ CLLE, Université de Toulouse, CNRS, UT2J, France. \\ ${ }^{\mathrm{c}}$ Institut Jean Nicod, CNRS UMR 8129, \\ DEC, ENS, PSL University, Paris, France.
}

\begin{abstract}
This paper aims at presenting a horizontal approach to the design of communication for joint action in human-robot interaction. According to this approach, social robotics must focus on different parameters of the whole joint action including context, the embedded situation and human psychological profile during the design and test process. Such an approach aims at complementing the standard building-block model that represents the state-of-the-art in robotic communication. Moreover, we provide some general ideas of how the model can facilitate the use of available communicative strategies for creating more efficient culturally sustainable robots in contexts of joint action.
\end{abstract}

Keywords. Communication, Joint Action, Building-block Model, Horizontal Model

\section{Introduction}

The advances of social robotics over recent decades raise several questions about their possible impact on human social practices as well as on human values such as integrity, respect, autonomy or freedom. For example, as Seibt et al. [1,2] emphasize, we know that humans seem to adapt to the linguistic abilities of robots [3] or that the kinematic profile of robots can trigger human mechanisms for motor contagion [4]; however, we don't know the long-term impact of these factors on social contexts shared with robots and humans. Furthermore, since, in principle, social robotics can exploit different uncontrollable human psychological mechanisms such as empathy [5], the technology could potentially undermine the user's autonomy, for example, her ability to interact with the robot according to her own reasons, wishes or preferences. Finally, the development of care and teaching robots for elderly citizens and children [6,7] raises important questions about the discharge of responsibility of caring for elders or

1 Corresponding Author: Víctor Fernández Castro. LAAS-CNRS, 7 Avenue du Colonel Roche, 31400 Toulouse; France. E-mail: vfernandezcastro@gmail.com

Acknowledgements: We would like to thank Nicola Butcher, Rachid Alami and Aurélie Clodic and two anonymous referees for their valuable comments and suggestions on earlier drafts of this paper. This research was supported by the Agence Nationale de la Recherche [Grant Number ANR-16-CE33-0017]. 
educating children in the hands of robots. It is indeed necessary to consider the value of human contact and the importance of the users' psychological health in these types of contexts. In this sense, a particularly relevant question is whether robots designed to carry out joint actions and collaborative tasks in the company of humans will be able to safeguard users' psychological integrity and dignity - e.g. not affecting their desires, emotions, preferences-without sacrificing functional efficiency. These types of questions not only serve as guidelines when addressing certain moral issues regarding social robotics but also help us to evaluate the current technical state of the art and the capacity of the discipline to enable robots with the necessary capabilities to carry out collaborative actions while avoiding potential harm to the users' psychological integrity and dignity.

Several authors have argued that, to properly address the uniqueness of humanrobot interaction, we must consider the interaction as a holistic and contextual experience and pay attention to how the robot meshes into the existing social structures and how it affects the context-dependency of the interactions [8-12]. So, one may argue that this approach may facilitate the evaluation of social robotics in joint action and its potential impact on human culture and values. Taking inspiration from this view, this paper aims to explore the consequences of a holistically inspired approach, which we coin the horizontal model, for communication in joint action for human-robot interaction (HRI). In particular, we will explore how attending to the contextual information, real objectives, preferences, expectations and other relevant parameters for the whole joint action achievement can change and improve the study of mechanisms for understanding and producing communicative signals in HRI.

To do so, we show that an important part of the current literature on communication in HRI reflects a particular approach [Section 2-3]. Such an approach, we coin the building-block model, face the design of communicative devices as isolated blocks, by abstracting it from the real context of the joint action. This model is the result of having a divide and conquer strategy and a manifestation of the global necessity of social robotics to investigate several isolated mechanisms before putting them to work into the wild. However, the building-block model, we believe, needs to be supplemented with a more general approach in order to be more efficient in the design of robust robots capable of navigating realistic settings and real-world environments. In particular, we argue that the horizontal model may provide a better insight on some important applications of communicative mechanisms [Section 4]. Finally, we explore the potential benefits of the model for designing more culturally and morally sustainable robots [Section 5].

\section{2. Robotic Communication for Joint Action}

Social robotics attempts to enable robots with different socio-cognitive capabilities for engaging and interacting with humans in different social environments [for a survey see 13 ] and tasks, including joint actions ${ }^{2}$. This requires both human and robot partners to

2 The capacity of the robots for doing or even simulating the capacities reviewed here are certainly limited. The present discussion is based on tests of software prototypes which are used in very specific and controlled situations or user studies based on "simulated" (scripted out using Wizard of Oz techniques) 
coordinate their actions and communicate properly in order to achieve a common goal. For example, the robot might detect the absence of its human partner or her inattention to provide her the information about what she missed without being annoying or intrusive [14]. Such capacity of analysis and communication helps to successfully and fluently execute the shared plan. Therefore, robots need to be endowed with both understanding and production of communicative signals in order to allow knowledge sharing, engagement maintenance or repair behaviors.

A representative example of HRI developments on communication is joint attention, which has focused especially on how the robot competences to follow human gazes or gestures positively impact the task performance and the human evaluation of the robot [15-18]. For instance, in a learning task the participants tended to detect quicker that an error would occur and addressed it when the robot used gaze cues to signal that it had finished its turn and was ready to engage in the next step of the task [19]. Besides collaborative performance, the use of non-verbal cues like gazes, facial expressions, lights or legible motions make the robot's internal states clearer for its human partner [20,21] and can serve to communicate its knowledge about the task execution state [22, 23]. For instance, the use of lights as indicators to communicate navigational intention has been shown to improve human understanding [23].

Another example is that different strategies have been deployed for robots to maintain the human engagement in collaborative tasks. Among them, a "highinteractivity behavior" such as greetings, use of gestures or lights, has been shown to increase the child engagement compared to a "low-interactivity" behavior [24]. In school-age children, curiosity can be seen as a key element to improve efficient learning [25], and recent studies have demonstrated that a robot exhibiting a curious behavior positively impacted children's curiosity and engagement in the task [26,27]. Finally, a robot adapting its behavior to its past experience with the user and establishing a friendly relationship by calling children by their names or confiding personal matters successfully led to maintaining a long-term interaction with children for two months [28].

Robotic communication also focuses on repairing errors that occur during the joint action. For instance, facial expressions in robots have been explored as feedback in order to orient human attention towards potential failures in the joint task [29, see also 30 for a review]. Moreover, some current research is attempting to handle failures by detecting human reactions such as head and shoulders movements [31] or gazes [32]. In a nutshell, we can find a large number of studies focused on developing different communication mechanisms to improve the joint action between robots and humans.

\section{The Building-Block Model}

The aforementioned studies are representative of a common and systematic way to approach the design of robotic capabilities that we coin the building-block model. As a first approximation, the model addresses a given capability or block in isolation from contextual aspects, concentrating on a canonical problem and testing the solution in

capabilities. 
controlled environments [13]. Such a way to proceed in the design of social robotics reflects the reasonable strategy of addressing the design of a particular block and testing its functioning by abstracting away different parameters and variables that may alter such functioning until the capacity has been proven to be robust enough. However, although necessary, we submit the idea that this building-block model needs to be supplemented for addressing the complexity of communication in social interaction. In this section, we emphasize different aspects of this model and indicate possible pitfalls that it should overcome when creating robots capable of operating in real environments

A first aspect is the contextual isolation of the investigated mechanism from the collaborative activity itself and its contextual parameters. An indicator of such isolation is the fact that many studies do not consider the final purpose or application in the design of the emotion recognition process [30]. For instance, emotion recognition may be oriented to identify a potential partner's failure during a joint action or to be used as an input for measuring the willingness to interact with the user. Another manifestation of the contextual isolation is that experimental settings and tasks are simple and unrealistic which gives a cue of the level of abstraction. To give an example, in Huang and Thomaz's [15] experiment, the setting aims to represent a learning task using a pretty simple labeling task without real objects and where the pointing gesture necessitates a pointer. Such contextual isolation may be necessary to develop the mechanisms in the first place-e.g. avoiding the potential problems of integrating the given mechanisms with other capacities. However, not having taken into account such contextual parameters may cause problems. For instance, in the emotion recognition process, a facial configuration like a smile may be interpreted as happiness or embarrassment depending on the context or the purpose of the mechanism. In the case of simplification of the task, one may obviate the fact that the meaning of pointing gestures or signals are strongly context-dependent and may affect the task (e.g. the learning tasks) in unexpected ways.

Secondly, building-block models often manifest an impersonal approach to communicative strategies which often uncouple human motivations, preferences, and goals from the joint action. This impersonal approach may overlook how these human motivations, preference and goals may vary from context to context, and thus, change the meaning of the communicative signals. For instance, attempting to exploit robot emotion expressions for maintaining engagement could be a functional strategy or not depending on the preferences of the users. For instance, some users may find it fun or interesting to interact with a more prosocial and engaging robot while others may find it intrusive and prefer a more discreet one. An example of the impersonal approach can be seen in Gordon and colleagues' study [26, see also 28] reviewed above, where they propose an engagement mechanism that is modulated by an intrinsic quasi-motivational mechanism which does not take human preferences or motivations into account. This type of mechanism can generate potential problems when tested in real environmentse.g. people do not always consider prosocial emotion expressions as appropriate. It can also produce unexpected unethical consequences [33] like emotional dependence in humans or exploiting their psychological vulnerability for engaging in interactions. 
Indeed, the robot is not able to produce the kind of reciprocity and balanced emotional feedback that one may obtain in real social interactions ${ }^{3}$.

The third aspect is the background isolation, which refers to the fact that developments in social robotics usually dissociate the mechanisms from background information that could be relevant for the joint action. Those background information include social norms, previous interactions with the partner, or cultural variability. To see an example, while an important part of the aforementioned studies concentrates on signaling or pointing [21,23], they abstract away part of the background information that could modulate the understanding of such signals $[34,35]$ which could dramatically change the meaning of the sign. For instance, we know that scrunching the nose in a particular direction may be seen as an expression of disgust for westerners but as a pointing gesture in other cultures [36].

Finally, the building-block model often concentrates on a very particular phase of joint action, obviating or not paying careful attention to the fact that joint action is a structured phenomenon which involves different stages or phases. In this respect, while HRI developments seem to put a lot of effort into solving particular communicative problems at the performance phase of the joint action, the initiation and the closing phase are not so well-developed and neither is how the different phases may impose different communicative and informational demands. Studies regarding engagement in HRI generally assume that humans will approach the robot if interested or they programmed the robot to search humans for engagement [37] but they overlook important informational parameters that should be established in the initiation phase and could dramatically change the communication strategies in the following phases. To see an example, consider Reyes and colleagues' studies [29]. As signaling anger could be an important sign of frustration and a signal that something could go wrong, such emotional response importantly depends on the relevant information exchanged in previous phases of the interaction. For example, if the initial phase of the interaction establishes different roles for the participants, e.g. a helper and a leader, the expression of frustration signaled by the leader may be interpreted as anger or a blaming reaction oriented to the helper; whereas it may be interpreted as being angry with oneself in a case in which it is previously established that participants have the same role . Again, abstracting away the relevant aspects of the environment is a productive strategy as a first step to generate and test reliable communicative mechanisms. However, to be able to integrate such mechanisms in a robot to enable it to navigate real social situations, new ways of approaching the different contextual, background and personal parameters of the joint actions where they are immersed must be considered.

\section{The Horizontal Model}

The building-block model above does not necessarily reflect a commitment or a consciously chosen procedure to design communicative strategies in joint action for

3 In Reyes and colleagues' studies [29], subjects reported to have made an effort for not offending the robot which indicates the emotional attachment that humans may establish with the robot and give a cue of their possible harming results for the users 
HRI. Instead, it reveals some features that the current state of the art in social robotics reflects. The necessity of complementing such a perspective with a more general view is not new [12]. In fact, the horizontal model we submit here is also supported in the emerging HRI literature, which emphasizes the need of focusing on how the specific robotic characteristics impact each or on the context-dependency of interactions $[8,11,12,38]$. Moreover, the proposed model can be understood as partially inspired by collaborative general frameworks that have been instantiated in the wild [39] and the literature in psychology and philosophy emphasizing the different levels of complexity involved in joint action and communication [40,41]. In this sense, our objective can be seen as a particular application of a widely held view to the specific challenge of communication in the context of joint actions for HRI.

The horizontal model can be introduced as the idea that communication in HRI joint action should not be abstracted away from the entire context and background where the particular collaborative task is embedded and situated in. Accordingly, the model needs to be horizontal in the sense that it should consider the joint action as a whole. It should also take into consideration how the different background information, the robotic and human capabilities and the context interact in complex ways with the communication devices in question. There are four basic aspects shaping the model.

The first aspect, contextualism, refers to the necessity of designing the proposed communicative device by taking into account the natural contexts (for both design and tests) where the collaborative task will take place. Indeed, effects of communicative strategies in a real-environment interaction highly depend on the environmental conditions. If the joint action takes place in a noisy spot (e.g. an airport or a shopping mall), verbal interactions can be hampered and should therefore be combined with other strategies in order to ensure the understanding of the human partner. Moreover, we know that communicative strategies are strongly context-dependent. Therefore, the same communicative signals, for instance opening eyes widely and raised eyebrows, can be an invitation to cooperate in certain contexts [42] while it could be interpreted as a sign of surprise in others [43]. As such, context-dependency may alter the intended meaning, especially in robots for which expressivity is often pretty limited [16]. However, the importance of context is not only revealed through how it alters the meaning of a particular signal or expression whose meaning is partially fixed but also through how it influences the function of the signals or expressions themselves. The function of certain types of signals are tied to the context of interaction or joint action and presuppose certain parameters, abilities or information that define their enable conditions. For instance, certain types of expressions like apologizing, offering or requesting help only carried out their communicative function in the context of joint action failures where one of the partners reacts to such failures with frustration or blaming expressions. In this sense, the function of these expressions cannot be performed if information like the acknowledgement of the failure or the reaction of the partner to this failure is missing. These cases reveal that context should not be considered after designing the communicative capacity to see how it influences the signals, but it must be included in the design process of the capacity itself from the very beginning, since the context shapes the function of the signal and communicative act itself. 
Secondly, the horizontal model must embrace a human-centered approach to put human preferences, values, and necessities at the center of the design process and test setting. This requires making the robot behavior transparent to the human partner at every stage. Indeed, the appropriate communication regarding capacities and preferences of each partner would generate coherent expectations, reduce uncertainties and increase predictability. Then, the robot could ask its human partner about her disabilities (e.g. disability to climb stairs, to see colors, etc.) and her preferences about a given set of parameters (e.g. speech volume, speech speed, language, etc.). For example, if in the initiation phase, the robot allows the human to set its speech volume, we avoid, here, misunderstandings in case of a human partner with hearing difficulties, as she will set the volume control high or, annoyance in case of a human partner with a sensitive ear as she will set the volume control low. Thus, it will improve fluidity during the joint action. Moreover, providing information to the human about upcoming action (moves) verbally or implicitly by exaggerating its movements, for instance, can avoid a feeling of insecurity or intimidation for the human because of the physical proximity of the robot.

Thirdly, the horizontal model must build its communication strategy as a situated capability which is embedded in a cultural and social space, and thus, must be sensitive to previous interactions, cultural variations and social norms. We have seen that proximal context can highly influence the interpretation of a particular communicative act like raising the eyebrows. However, previous interactions or social norms can equally impact communicative acts. For instance, from early infancy, humans are able to interpret differently the same act of pointing depending on the common ground they share with an adult [44]. If a pointing gesture is carried out in an interaction where the child was previously doing a particular related object task, the act is interpreted as a guide (now, pick this object). However, if the common ground with the adult is absent, the pointing gesture is interpreted as a demand (give me the object). Such variation demonstrates that communicative actions are modulated by previous interactions. Moreover, some fundamental elements of prediction and coordination in joint action are available thanks to a rich array of background information like social norms, convention or scripts regulating our social interactions [45, for a perspective from philosophy of social robotics see 46,47]. Such norms can, for instance, establish different roles during the interaction. Hence, communication parameters could be modulated such as a service robot dealing with customers and colleagues must be able to display different communicative strategies depending on the role of the human partner.

Finally, the horizontal model recognizes joint action as a whole. As we saw in section 3 , the production of a given signal must be affected by knowledge established in previous stages of the joint action (e.g. whether the roles of the partner have been established). Therefore, the studied communicative devices must be responsive to the potential variation of information that depends on stages of the joint action that precede the production of the signal. Indeed, joint action requires the two partners to plan and perform their actions according to the common goal they determined at the very beginning of the interaction [48]. They also need to predict the other's actions in order to reach this goal in an effective way for both partners [49]. It is thus necessary to integrate the different stages of the joint action, its whole process, from its initiation to 
its closure. For this, communicative strategies must be used in order to add, modify and update the common ground associated with the joint task. Operating in a horizontal model of the joint action and so considering its complete process, firstly involves the sharing of knowledge between the two partners during the initiation phase of the joint action (roles, common goal, sub-goals, individual action plans, expectations, or capacities). Different communicative strategies can be used for different purposes according to the stage of the joint action. For example, using emotional signals like surprise when the robot fails to recognize what the human is doing can be used to precisely indicate this inability to the human, but also as an anticipatory signal of a rectification, a re-planning or an invitation to let the human taking care of this sub-task. In addition, Breazeal and colleagues [19] showed that gaze signals from the robot when it finishes its turn and then communicates that it is ready for the next step improve the human understanding. It also allows the human to anticipate and address potential errors in the task. Equipping a robot with such capabilities during the whole joint action would allow it to communicate its readiness and engagement to the task and would consequently help to decrease uncertainties. Indeed, these communicative eye signals can be seen as a form of commitment for the joint action and so for initiating it [42]. Finally, it would produce quicker reactions from the human if something goes wrong and help to disambiguate the situation [50].

\section{Towards Culturally Sustainable Robotics}

One of the main implications of the horizontal model is that the communicative mechanisms should be integrated with other mechanisms or blocks, especially those like perception or planning mechanisms that facilitate the recognition and reasoning regarding the environment and the human actions and mind. As such the components can nourish one another and improve the overall interaction. In this sense, a horizontal perspective provides the capacity to confront aspects of the joint action that, otherwise, would perhaps go unnoticed. However, we would like to sketch another consequence of the horizontal model, namely how it may promote the design of robots to be as minimally invasive as possible with human values, feelings, or preferences, and to be respective of human integrity. The first reason is, of course, its human-centered approach. Considering the human partner at the center of the interaction does not only mean to consider her reactions but considering the probably unexpected harmful consequences of the robot behavior. In this regard, Brinck and Balkenius [33] have argued that the widely spread strategy of boosting emotional responses in humans raises ethical concerns as much as social robots aiming at exploiting the emotional vulnerability of human users. In the horizontal model, considerations regarding the potential damaging or unethical consequences of using, for instance emotion expressions, can motivate different solutions. Therefore, the robot could facilitate the same task by using other communicative strategies. To give an example, robots could exploit different communicative signals to indicate errors in the joint action or to express concern about the human goals without necessarily using emotion expression, for instance using emotionally neutral but recognizable signals [51]. 
Secondly, we know that every joint action is at risk of suffering different types of uncertainties [52], so there is no way to calculate all the potential failures and consequences of human-robot interaction. However, as we have sketched, when we are able to integrate our communicative strategies in their context and social situation, we can use different communicative strategies to try to repair and rectify when a problem, failure or potential moral harm has occurred-e.g. providing justifications or apologizing. As such, developing robotic agents capable of repairing their potential moral damage or their failure to respect the cultural integrity of an agent requires a horizontal perspective that gives us a broad vision when integrating and contextualizing regulatory and reparatory communication strategies [53].

\section{Conclusion}

This paper aims to show the need to complement the standard proposal concerning the design of communication in the joint action for human-robots interaction with a more horizontal vision. This perspective can help us to create more efficient social robots from a functional point of view but also explore different ways to create less invasive robots which integrate human being as a social and cultural agent. In this sense, despite the progress of the building-block strategy to acquire a large number of potentially interesting tools, a complementary model that facilitates the process to deal with real contexts of collaboration with humans is necessary. If we want to advance in creating social robots that do not negatively impact the values and integrity of their human partners, we must start to consider a horizontal strategy to complement their design.

\section{References}

[1] Seibt J. "Integrative Social Robotics": A new method paradigm to solve the description problem and the regulation problem? In: Seibt J, Nørskov M, Schack Andersen S, red., What social robots can and should do: Proceedings of Robophilosophy 2016/TRANSOR 2016. Amsterdam: IOS Press. 2016.

[2] Seibt J, Damholdt MF, Vestergaard C. Five Principles of Integrative Social Robotics. In: Coeckelbergh M, Loh J, Funk M, Seibt J, Nørskov M, editors, Envisioning Robots in Society-Power, Politics, and Public Space: Proceedings of Robophilosophy 2018. Amsterdam : IOS Press. 2018.

[3] Fischer K. The role of users' preconceptions in talking to computers and robots. In: Fisher K, editor, How people talk to computers, robots, and other artificial communication partner: Proceedings of the Workshop Hansewissenschaftskolleg. Delmenhorst. 2006.

[4] Bisio A, Sciutti A, Nori F, Metta G, Fadiga L, Sandini G,et al. Motor contagion during human-human and human-robot interaction, PLoS One. 2014;9(8).

[5] Kwak SS, Kim Y, Kim E, Shin C, Cho K. What makes people empathize with an emotional robot? The impact of agency and physical embodiment on human empathy for a robot. In: The $22^{\text {nd }}$ IEEE International Symposium on Robot and Human Interactive Communication, IEEE RO-MAN. 2013. p. 180-185.

[6] University of Southern Denmark, SDU [Internet]. Odense(Denmark): SDU Robotics; 2020. SMOOTHSeamless human-robot interactiOn fOr THe support of elderly people [updated 24.07.2020; cited 01.09.2020]. Available from: https://www.sdu.dk/en/om sdu/institutter centre/sdurobotics/researchprojects/smooth.

[7] MIT media lab [Internet]. Cambridge, MA(USA): Massachusetts Institute of Technology;[date unknown]. Huggable: A social robot for pediatric care [cited 01.09.2020]. Available from: https://www.media.mit.edu/projects/huggable-a-social-robot-for-pediatric-care/overview/ 
[8] Cramer H, Goddijn J, Wielinga B, Evers V. Effects of (in)accurate empathy and situational valence on attitudes towards robots. In: The 5th ACM/IEEE International Conference on Human-Robot Interaction (HRI). 2010. p. 141-142.

[9] Milliez G, Warnier M, Clodic A, Alami R. A framework for endowing an interactive robot with reasoning capabilities about perspective-taking and belief management. In: The 23rd IEEE International Symposium on Robot and Human Interactive Communication. 2014. p. 1103-9.

[10] Menezes P, Quintas J, Miranda Dias J. The Role of Context Information in Human-Robot Interaction. In: The 23rd IEEE International Symposium on Robot and Human Interactive Communication, Workshop on Interactive Robots for aging and/or impaired people. 2014.

[11] Young JE, Sung J, Voida A, Sharlin E, Igarashi T, Christensen HI, et al. Evaluating Human-Robot Interaction: Focusing on the Holistic Interaction Experience. Int J Soc Robot. 2011 Jan;3(1):53-67.

[12] Seibt J, Damholdt MF, Vestergaard C. Integrative social robotics, value-driven design, and transdisciplinarity. Interact Stud Soc Behav Commun Biol Artif Syst. 2020;21(1):111-44.

[13] Thomaz A, Hoffman G, Cakmak M. Computational Human-Robot Interaction. Found Trends Robot. 2016;4(2-3):104-223.

[14] Devin S, Alami R. An Implemented Theory of Mind to Improve Human-Robot Shared Plans Execution. In: Proceedings of the 11th ACM/IEEE International Conference on Human Robot Interaction HRI '16. Christchurch, New Zealand: IEEE Press; 2016. p.319-326.

[15] Huang CM, Thomaz AL. Joint Attention in Human-Robot Interaction. In: AAAI Fall Symposium: Dialog with Robots. 2010.

[16] Admoni H, Scassellati B. Social Eye Gaze in Human-Robot Interaction: A Review. J Hum-Robot Interact. 2017;6(1).

[17] Boucher J-D, Pattacini U, Lelong A, Bailly G, Elisei F, Fagel S, et al. I Reach Faster When I See You Look: Gaze Effects in Human-Human and Human-Robot Face-to-Face Cooperation. Front Neurorobotics . 2012;6.

[18] Staudte M, Crocker MW. Visual attention in spoken human-robot interaction. In: Proceedings of the 4th ACM/IEEE international conference on Human robot interaction - HRI '09. La Jolla, California, USA: ACM Press; 2009.

[19] Breazeal C, Kidd CD, Thomaz AL, Hoffman G, Berlin M. Effects of nonverbal communication on efficiency and robustness in human-robot teamwork. In: Proceedings of IEEE/RSJ International Conference on Intelligent Robots and Systems (IROS 2005). 2005. p. 708-713.

[20] Sisbot EA, Alami R. A human-aware manipulation planner. IEEE Transactions on Robotics. 2012;28(5):1045-1057.

[21] Breazeal C. Function Meets Style: Insights From Emotion Theory Applied to HRI. IEEE Trans Syst Man Cybern Part C Appl Rev. 2004;34(2):187-94.

[22] Clodic A, Cao H, Alili S, Montreuil V, Alami R, Chatila R. SHARY: A Supervision System Adapted to Human-Robot Interaction. In: Khatib O, Kumar V, Pappas GJ, editors. Experimental Robotics. Berlin, Heidelberg: Springer; 2009:229-38. (Springer Tracts in Advanced Robotics).

[23] May AD, Dondrup C, Hanheide M. Show me your moves! Conveying navigation intention of a mobile robot to humans. In: Proceedings of the European Conference on Mobile Robots (ECMR). 2015. p. 16.

[24] Tozadore DC, Pinto AHM, Romero RAF. Variation in a Humanoid Robot Behavior to Analyse Interaction Quality in Pedagogical Sessions with Children. In: 2016 XIII Latin American Robotics Symposium and IV Brazilian Robotics Symposium (LARS/SBR) . Recife, Brazil: IEEE; 2016. p. 1338.

[25] Arnone MP, Small RV, Chauncey SA, McKenna HP. Curiosity, interest and engagement in technologypervasive learning environments: a new research agenda. Educ Technol Res Dev. 2011;59(2):181-98.

[26] Gordon G, Breazeal C, Engel S. Can Children Catch Curiosity from a Social Robot? In: Proceedings of the Tenth Annual ACM/IEEE International Conference on Human-Robot Interaction - HRI '15 . Portland, Oregon, USA: ACM Press; 2015. p. 91-98.

[27] Ceha J, Chhibber N, Goh J, McDonald C, Oudeyer P-Y, Kulić D, et al. Expression of Curiosity in Social Robots: Design, Perception, and Effects on Behaviour. In: Proceedings of the 2019 CHI Conference on Human Factors in Computing Systems - CHI '19. Glasgow, Scotland Uk: ACM Press; 2019. p. 1-12.

[28] Kanda T, Sato R, Saiwaki N, Ishiguro H. A Two-Month Field Trial in an Elementary School for LongTerm Human-Robot Interaction. IEEE Trans Robot. 2007 Oct;23(5):962-71. 
[29] Reyes M, Meza I, Pineda LA. The Positive Effect of Negative Feedback in HRI Using a Facial Expression Robot. In: Koh JTKV, Dunstan BJ, Silvera-Tawil D, Velonaki M, editors. Cultural Robotics. Cham: Springer International Publishing; 2016. p. 44-54. (Lecture Notes in Computer Science).

[30] Cavallo F, Semeraro F, Fiorini L, Magyar G, Sinčák P, Dario P. Emotion Modelling for Social Robotics Applications: A Review. J Bionic Eng. 2018;15(2):185-203.

[31] Trung P, Giuliani M, Miksch M, Stollnberger G, Stadler S, Mirnig N, et al. Head and shoulders: automatic error detection in human-robot interaction. In: Proceedings of the 19th ACM International Conference on Multimodal Interaction - ICMI 2017. Glasgow, UK: ACM Press; 2017. p. 181-8.

[32] Aronson RM, Admoni H. Gaze for Error Detection During Human-Robot Shared Manipulation. In: Workshop Towards a Framework for Joint Action at Robotics: Science and Systems (RSS2018). 2018.

[33] Brinck I, Balkenius C. Mutual Recognition in Human-Robot Interaction: a Deflationary Account. Philos Technol. 2018;33(1):53-70.

[34] Liebal K, Colombi C, Rogers SJ, Warneken F, Tomasello M. Helping and Cooperation in Children with Autism. J Autism Dev Disord. 2008 Feb;38(2):224-38.

[35] Moll H, Tomasello M. Level 1 perspective-taking at 24 months of age. $\mathrm{Br} \mathrm{J}$ Dev Psychol. 2006;24(3):603-13.

[36] Cooperrider K, Slotta J,Núñez R. The Preference for Pointing With the Hand Is Not Universal. Cogn Sci, 2018;42:1375-1390.

[37] Finke M, Kheng Lee Koay, Dautenhahn K, Nehaniv CL, Walters ML, Saunders J. Hey, I'm over here How can a robot attract people's attention? In: ROMAN 2005 IEEE International Conference on Robot and Human Interactive Communication, 2005. Nashville, TN, USA: IEEE; 2005. p. 7-12.

[38] Clodic A, Pacherie E, Alami R, Chatila R. Key Elements for Human-Robot Joint Action. In: Hakli R, Seibt J, editors. Sociality and Normativity for Robots. Cham: Springer International Publishing; 2017. p. 159-77. (Studies in the Philosophy of Sociality) .

[39] Clodic A, Fleury S, Alami R, Chatila R, Bailly G, Brethes L, et al. Rackham: An Interactive RobotGuide. In: ROMAN 2006 - The 15th IEEE International Symposium on Robot and Human Interactive Communication. 2006. p. 502-9.

[40] Pacherie E. The Phenomenology of Joint Action: Self-Agency vs. Joint-Agency. Seemann, A. editor. Joint Attention: New Developments, MIT Press; 2012. p.343-389.

[41] Clark H. Using language. Cambridge: Cambridge University Press. 1996

[42] Siposova B, Tomasello M, Carpenter M. Communicative eye contact signals a commitment to cooperate for young children. Cognition. 2018;179:192-201.

[43] Noordweir M, van Dijk E. Surprise: unfolding of facial expressions. Cognition and Emotion. 2019;33(5):915-30.

[44] Liebal K, Behne T, Carpenter M, Tomasello M. Infants use shared experience to interpret pointing gestures. Dev Sci. 2009;12(2):264-71.

[45] Zawidzki T. Mindshaping: a new framework for understanding human social cognition. Cambridge, Mass: MIT Press; 2013.

[46] Fernandez Castro V. Shaping Robotic Minds. In: Sociable Robots and the Future of Social Relations: Proceedings of Robo-Philosophy 2014. IOS Press; 2014

[47] Fernandez Castro V. Mindshaping and Robotics. In: Hakli R, Seibt J, editors. Sociality and Normativity for Robots: Philosophical Inquiries into Human-Robot Interactions. Cham: Springer International Publishing; 2017. p. 115-35. (Studies in the Philosophy of Sociality).

[48] Knoblich G, Butterfill S, Sebanz N. Psychological Research on Joint Action. In: Psychology of Learning and Motivation. Elsevier; 201. p. 59-101.

[49] Sebanz N, Knoblich G. Prediction in Joint Action: What, When, and Where. Top Cogn Sci. 2009 Apr;1(2):353-67.

[50] Admoni H, Dragan A, Srinivasa SS, Scassellati B. Deliberate delays during robot-to-human handovers improve compliance with gaze communication. In: Proceedings of the 2014 ACM/IEEE international conference on Human-robot interaction - HRI '14. Bielefeld, Germany: ACM Press; 2014. p. 49-56.

[51] Baraka K, Alves-Oliveira P, Ribeiro T. An extended framework for characterizing social robots. In: Jost C., et al., editors. Human-Robot Interaction, Springer Series on Bio- and Neurosystems. Springer, Cham. 2020;12.

[52] Michael J, Pacherie E. On Commitments and Other Uncertainty Reduction Tools in Joint Action. J Soc Ontol. 2015;1(1):89-120. 
[53] Malle B, Scheutz M. "When will people regard robots as morally competent social partners?." 2015 24th IEEE International Symposium on Robot and Human Interactive Communication (RO-MAN). IEEE, 2015. 